\title{
Association of Hepatitis C with Hepatocelluar Carcinoma: A Cross-Sectional Study from Northern Pakistan
}

Keywords: Hepatocellular carcinoma; Hepatitis C; Risk factor, Abdominal pain; J aundice

\begin{abstract}
Aim: Hepatocellular Carcinoma (HCC) is one of the most deleterious among cancer types which is associated with high morbidity and mortality. The disease more commonly affects men usually in their fifth and sixth decade of life. Hepatitis $C$ is considered to be the most common risk factor associated with hepatocellular carcinoma. The aim of our study was to determine the association of Hepatitis C with HCC among patients presenting at our hospital from Northem Pakistan and impact on management and prognosis.
\end{abstract}

Materials and methods: A cross sectional study that was caried out overa period of one and half years included a total of 145 patients of hepatocellularcarcinoma. The data wasassessed and viral serology was done for all the patients. Analysis was done using SPSS version 22.

Results: Seventy three percent patients in our study group were positive for HepatitisC. Most patients presented in late stageswith poor perfomance status in the background of severe chronic liver disease and cimhosis. Most common presenting symptoms were abdomina pain, distension and jaundice. Most patients were subjected to palliative and best supportive care and there was no candidate for curative surgic al treatment.

Conclusion: The study concluded that Hepatitis C associated HCC is most common in our area yet the major burden of the disease is remains undetected due to lack of a wareness and national screening programs leading to late presentation with incurable disease.

\section{Objective}

To determine the association of Hepatitis $\mathrm{C}$ virus as a risk factor in patients of hepatocellular carcinoma and to see the relationship of Hepatitis C positivity with prognosis.

\section{Materials and Methods}

Study Design: Descriptive cross sectional study.

Setting: Atomic Energy Cancer Hospital (AECH) NORI.

Duration of the Study: From January, 2017 to July, 2018.

Sampling technique: Non-probability consecutive sampling.

\section{Data Collection}

The study was approved by hospital Ethical Review Committee (ERC) and research training and monitoring cell (RTMC). Informed consent was taken from the patients included in the study and fulfilling the inclusion criteria. Patients included in the study were those registered at outpatient oncology department of $\mathrm{AECH}$, NORI. They were evaluated in detail by history, general and systemic

\section{Journal of}

\section{Cancer Sciences}

\author{
Fatima $\mathrm{H}^{1^{\star}}$, Mahmood $\mathrm{H}^{2}$ and Faheem $\mathrm{M}^{3}$ \\ ${ }^{1}$ Resident Oncology, Atomic Energy Cancer Hospital, NORI \\ ${ }^{2}$ Head of Department Oncology, Atomic Energy Cancer Hospital, NORI \\ ${ }^{3}$ Chief Oncologist and Director, Atomic Energy Cancer Hospital, NORI \\ *Address for Correspondence \\ Fatima H, Resident Oncology, Atomic Energy Cancer Hospital, NORI, \\ Pakistan, E-mail: hadia.fatima@hotmail.com \\ Submission: 20 November, 2018 \\ Accepted: 24 December, 2018 \\ Published: 26 December, 2018 \\ Copyright: @ 2018 Fatima H. This is an open access article distributed \\ under the Creative Commons Attribution License, which permits \\ unrestricted use, distribution, and reproduction in any medium, provided \\ the original work is properly cited.
}

physical examination and investigations including hematology, biochemistry and radiological studies. The data were recorded in terms of demographic profile, patient's presentation, performance status in form of ECOG score, viral serology for which Enzyme Linked Immunosorbent Assay (ELISA) was done to detect Anti-IgM $\mathrm{HCV}$ antibody and HbsAg antibody for the presence of Hepatitis $\mathrm{C}$ and $\mathrm{B}$ virus, alpha fetoprotein levels, Child-Pugh class and TNM stage of the disease.

\section{Data Analysis}

The collected data were entered and analyzed using SPSS V 22. Descriptive statistics were calculated for both qualitative and quantitative variables. Qualitative variables were presented as percentages/frequency. Quantitative variables including age were presented by mean $+\mathrm{SD}$. Age and stage of the disease was controlled by stratification. Post stratification chi-square test was applied and $p$ value $<0.05$ was considered significant.

\section{Introduction}

Hepatocellular carcinoma is a primary liver malignancy originating from hepatic stem cells. It mostly occurs in the background of chronic liver disease and cirrhosis [1]. Macroscopically the tumor can be infiltrating or nodular [1]. Microscopically ranges from well differentiated or poorly differentiated forms [1].

HCC is the sixth most common malignancy and the third leading cause of cancer related death worldwide [1]. Over the last two decades the incidence of HCC has almost doubled worldwide and Hepatitis C is considered to be the fastest growing cause of overall mortality and hence termed as an "Epidemic". The highest prevalence of HCC is in East Asia followed by Africa. In Pakistan, frequency of Hepatitis C in blood donors is $3 \%$ and $4.7 \%$ in general population, much higher than in neighboring countries [2-4].

The common risk factors attributing to hepatocellular carcinoma include Hepatitis C infection, Hepatitis B infection, alcoholic cirrhosis, tobacco, Oral contraceptive pills, aflatoxin exposure and hemochromatosis [1]. In Pakistan, approximately $2.4 \%$ healthy adult population is infected with hepatitis B and 3.0\% with hepatitis C virus with much higher prevalence among high risk groups [5]. In 
Citation: Fatima H, Mahmood H, Faheem M. Association of Hepatitis C with Hepatocelluar Carcinoma: A Cross-Sectional Study from Northern Pakistan. J Cancer Sci. 2018;5(1): 5

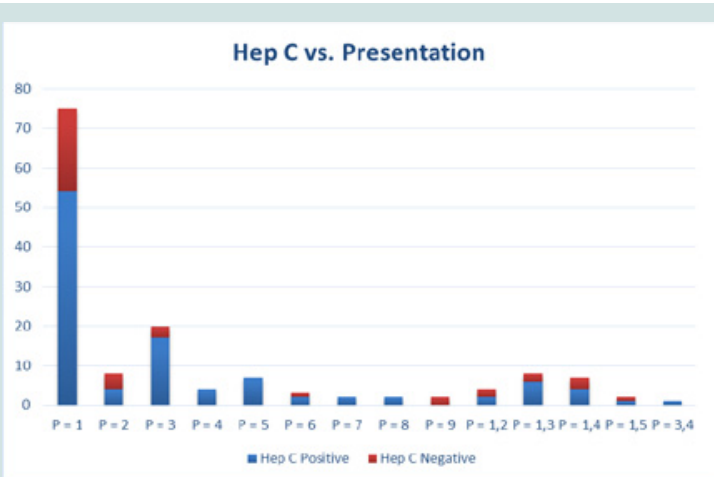

Figure 1: Association between Hepatitis $C$ and clinical presentation of the HCC patients.

Clinical Presentation: P1: Abdominal Pain; P2: Abdominal Distension; P3: Jaundice; P4: Anorexia; P5: Hematemesis; P6: Brain metastasis; P7: Cord compression; P8: Weightloss; P9: Bone Pains.

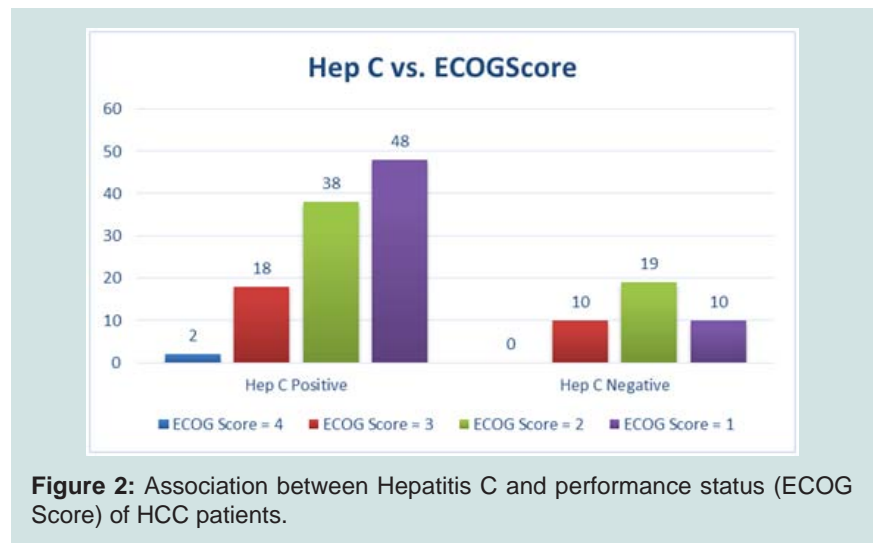

the developing countries Hepatitis B is considered to be the most important cause of HCC but HCV infection is most commonly involved in pathogenesis of about $60-70 \%$ patients of HCC in groups [5]. The aim of our study was to evaluate the role of hepatitis $C$ virus as a risk factor in the development of hepatocellular carcinoma in our population. This would help giving recommendations in future to the clinical oncologists and help predicting the outcome of the disease process.

\section{Results}

A total of 145 patients presented at Atomic Energy Cancer Hospital (AECH), NORI with the diagnosis of hepatocellular carcinoma from January, 2017 to July, 2018. Out of 145 patients $70.3 \%$ were male and $29.7 \%$ were female. Mean age of the patients was 59.68 years \pm 10.58 years. Mean age of the male patients was $58.67 \pm 10.4$ years and female patients was $62.02 \pm 10.7$ years. A total of $73.1 \%$ patients were positive for Anti-HCV IgM antibody while $26.9 \%$ patients were negative. Only $3.4 \%$ patients were positive for $\mathrm{HBsAg}$ antibody while only $1.3 \%$ patients had HCV and HBV coinfection. The chief presenting complaint in most of the patients was abdominal pain (51.7\%), followed by jaundice (13.8\%) and abdominal distension (5.5\%). While patients also presented with vomiting and hematemesis (4.8\%), weight loss (1.4\%), anorexia (2.8\%), bone pains (1.4\%), spinal cord compression (1.4\%) and brain metastasis (2.1\%) and multiple signs and symptoms (15.2\%). P-value is 0.174 (Figure 1).
Regarding performance scoring $40 \%$ patients were ECOG 1, $39.3 \%$ were ECOG 2, 19.3\% were ECOG 3 and 1.4\% patients were ECOG 4 at the time of presentation (P-value 0.122). Child-Pugh's scoring was done for all the patients. $22.8 \%$ had Child class A, $45.5 \%$ had Child class B and $31.7 \%$ had Child class $\mathrm{C}$ at the time of presentation (P-value 0.157) (Figure 2 and 3).

$86.2 \%$ patients had no family history of any malignancy while only $13.8 \%$ patients had positive family history of malignancy. AFP levels of one hundred and ten patients was available with mean value of $12125.1 \pm 3064.8 \mathrm{ng} / \mathrm{ml}$, which were categorized in 5 groups for prognostic evaluation (P-value 0.254) (Figure 4).

TNM staging was done for all the patients showed that $58.6 \%$ had stage IV disease at the time of initial presentation, $27.6 \%$ had stage III, $12.4 \%$ had stage II and $1.4 \%$ had stage I hepatocellular carcinoma (P-value 0.733) (Figure 5).

$62.8 \%$ patients had no associated comorbids, $9.7 \%$ patients had hypertension, $11 \%$ were diabetic, $0.7 \%$ patient had ischemic heart disease, $2.1 \%$ patients had asthma, $2.9 \%$ had history of pulmonary tuberculosis, and $0.7 \%$ had second malignancy while $7.6 \%$ patients had multiple comorbids. The survival outcome was very poor in these patients and moratality rate was almost $100 \%$ at 1 year. None of the patients had benefited much from systemic treatment. Recurrence rate was high for those early stage patients who had undergone TACE and RFA. No patient was a candidate for curative surgical resection

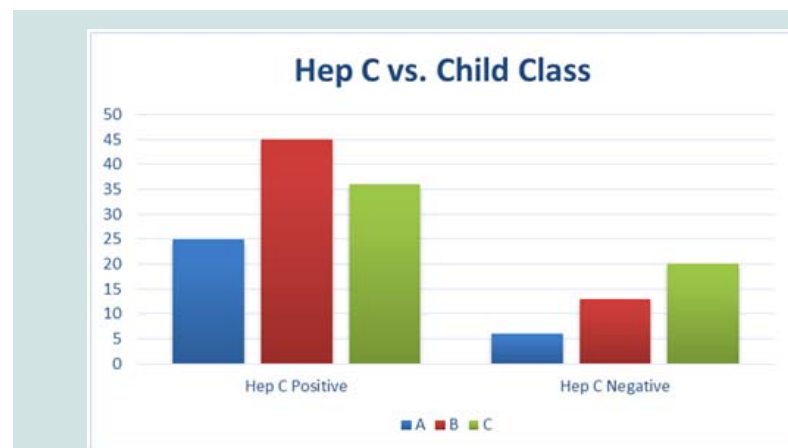

Figure 3: Association between Hepatitis C and Child Pugh class of HCC patients.

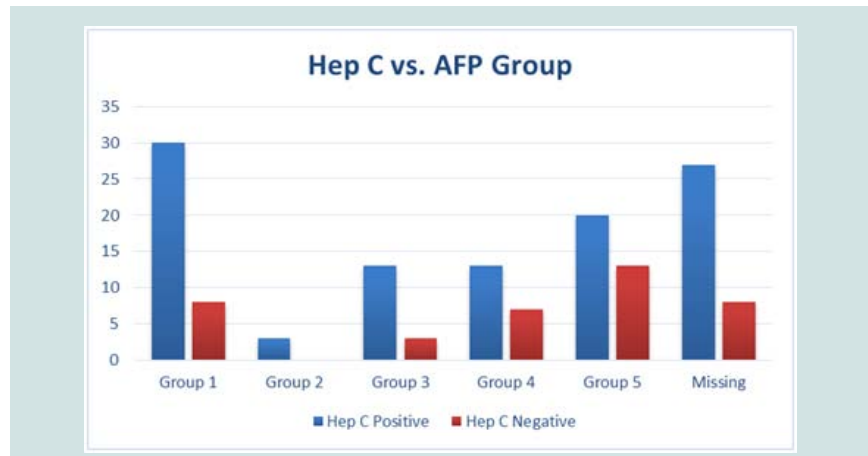

Figure 4: Association between Hepatitis C and Alpha Fetoprotein levels of HCC patients.

AFP Levels: Group 1: 0-50 ng/ml; Group 2: 51-100 ng/ml; Group 3: 101-500 ng/ml; Group 4: 501-5000 ng/ml; Group 5: > 5000 ng/ml. 


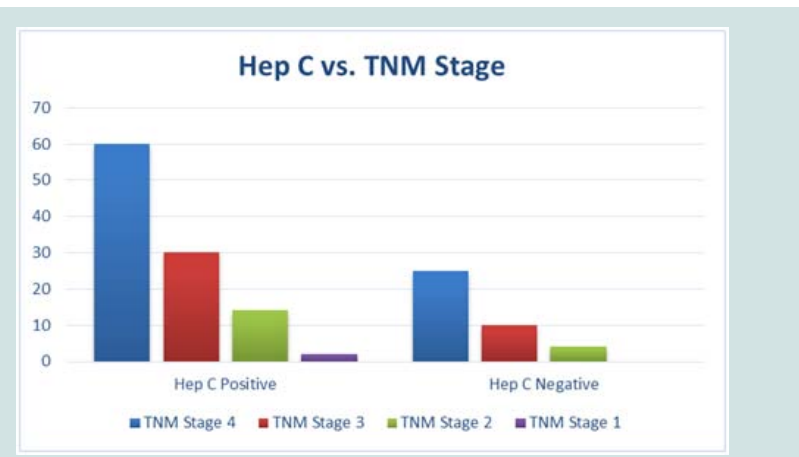

Figure 5: Association between Hepatitis C and TNM stage of HCC patient.

and liver transplant.

\section{Discussion}

HCC accounts for up to $90 \%$ primary liver malignancies [7]. Hepatocellular carcinoma is an extremely lethal disease as its mortality rate is almost $100 \%$ i.e the incidence rate equals mortality rate [3]. Due to the poor survival rate of approximately $5 \%$, only few patients tend to receive radical treatment [3]. More than $80 \%$ of the cases are reported from developing countries [6]. HCC is preceded by chronic liver inflammation which can occur possibly in the background of mainly chronic viral infections, toxins, autoimmune disorders and metabolic syndromes [1]. In Eastern Asia the most common risk factors are hepatitis $\mathrm{B}$ and $\mathrm{C}$ infection and aflatoxin exposure [8].

The risk of HCC in patients with Hepatitis $\mathrm{C}$ infection increases up to 17 fold [9]. Annual incidence is $2-6 \%$ which may take up to two decades to develop [9]. Hepatocarcinogenesis is a multistep process and there is slow accumulation of genetic mutations [9]. According to Pakistani statistics, $58 \%$ of the reported cases of HCC are Hepatitis C positive and $25.3 \%$ are positive for Hepatitis B [6]. In our study the frequency of HCV positive HCC was much higher i.e., 70.3\% while only $3.4 \%$ patients were positive for Hepatitis B. Although the statistics may vary in different parts of the country as our study is limited to single institution and no national cancer registry statistics are available. There is no such parameter available through which we can do risk evaluation and casualty assessment in HCV positive HCC patients. There is marked demographic variation among different populations. HCC is common among male population, and among the most common malignancies among adult males in Pakistan. Overall incidence ratio among male to female is $3: 1$ with shifting trends towards younger population 3 . In our study $70.3 \%$ patients were male and $29.7 \%$ were females making the incidence radio of 2.37:1 in our population. The majority of our patients presented in $5^{\text {th }}$ and $6^{\text {th }}$ decade of life. In our study one patient of 17 years of age was also enrolled who had presented with late stage HCC.

HCC remains latent for 3.2 years [6]. It is very difficult to differentiate the diffuse tumors from the cirrhotic liver tissue but the early diagnosis during the subclinical period has a propensity to cure the disease [6]. Screening is usually done through ultrasound along with measurement of serum levels of Alpha Fetoprotein (AFP) [6]. Asian Oncology Summit recommendations for screening are 6 monthly ultrasound plus measurement of serum AFP levels [10]. The sensitivity of ultrasound increases up to $79 \%$ when correlated with AFP levels [11]. As compared to the western world where there are well established programs for screening of high risk patients of chronic liver disease, HCC is detected in earlier stages, while only up to $10 \%$ patients in Pakistan undergo routine screening tests [6]. Unfortunately none of the patients enrolled in our study underwent HCC screening and were detected incidentally when became symptomatic for the disease. At presentation majority of the patients had raised AFP levels in our study group. Patients of HCC may remain asymptomatic for long periods of time or may present with chronic liver disease. Majority of the patients had not received any systemic treatment for HCV or had poor compliance. Clinical presentation may vary from patient to patient. In our study most common presenting symptoms were abdominal pain and jaundice.

Contrast enhanced Multiphasic MRI and CT scan of liver is considered to be the classic imaging modality to make the definitive diagnosis of HCC [6]. A meta-analysis showed that USG is although less sensitive but most specific up to $97 \%$. Tumor marker AFP is advised along with imaging studies to reach to the final diagnosis. Elevated AFP levels of $>400 \mathrm{ng} / \mathrm{ml}$ is associated with active HCC. Biopsy is only indicated in HCC when there are suspicious nodules or suspicion of some other malignancy [6]. Biopsy is also indicated only when serum CA19-9 and CEA levels are raised and cholangiocarcinoma needs to be ruled out [13]. In our study the diagnosis was made through the combination of imaging via Triphasic CT scan and serum AFP levels in all the patients. Biopsy was not advised to any of our patients. Most of the patients in our setup presented in the advanced stages and markedly elevated AFP levels associated with HCV positivity.

To find out the extent of the disease many staging systems have been developed in clinical practice [14]. AJCC TNM staging system is employed in our setup to find out the extent of HCC. It includes the standard method of tumor, node and metastasis evaluation and is a predictor of prognosis and treatment outcome. In Pakistan up to $62 \%$ patients present with multifocal HCC and $44.3 \%$ patients present with large tumor sizes of $>5 \mathrm{~cm}$ [6]. In our study $58.6 \%$ patients presented with multifocal or large space occupying liver lesions stage IV disease, followed by $27.3 \%$ patients with stage III disease. $12.4 \%$ patients had stage II disease and only $1.2 \%$ had stage I HCC. HCV+ patients presented in more advanced stages as compared to HCVpatients and had Child-Pugh class B and C according to Child-Pugh classification system.

Ideally all cases of HCC should be discussed in multidisciplinary tumor board to reach upon a treatment plan. It is a dilemma that 'Cure' is usually not achieved in Hepatocellular carcinoma and there is no single treatment option which has been agreed upon in this tumor. Most of the patients in our study were subjected to palliative treatments after initial evaluation and discussion in tumor board. The treatment options are potentially curative, palliative and supportive and none of them is considered definite therapy [15]. The potentially curable treatment options include surgical resection, liver transplant and radiofrequency ablation which are usually offered to the early stage HCC patients [15]. Surgical resection is considered to be the mainstay of treatment in patients with good performance status and Child class A having very early stage solitary tumors with no cirrhosis and portal hypertension [15]. Only 5-10\% patients are considered fit for surgery with five year overall survival and recurrence rate up to 
Citation: Fatima H, Mahmood H, Faheem M. Association of Hepatitis C with Hepatocelluar Carcinoma: A Cross-Sectional Study from Northern Pakistan. J Cancer Sci. 2018;5(1): 5

$70 \%$ and $68 \%$ respectively in HCC patients [15]. None of our study group patients was amenable for surgical resection. Referring to the Milan criteria solitary tumor of $<5 \mathrm{~cm}$ or up to 3 tumors of $<3 \mathrm{~cm}$ are considered for liver transplant [15]. Active Hepatitis C infection is likely to cause tumor recurrence in up to $40 \%$ of the liver transplant patients [16]. Other poor prognostic features likely to cause recurrence include multilobar disease, macroscopic vascular invasion, high grade tumor and pretreatment AFP levels of $>300 \mathrm{ng} / \mathrm{ml}$ [17]. The lack of good quality donor organs and expertise, liver transplant is a very limited treatment approach while other alternative approaches are usually considered in this regard. None of the patients in our study was offered liver transplant.

Another well-defined treatment approach in HCC is Radiofrequency Ablation RFA. It is an ablative procedure and is indicated in small well defined tumors of $<5 \mathrm{~cm}$ and has best results when tumor size is $<2 \mathrm{~cm} \mathrm{18}$. Five year overall survival rate is from 40 to $70 \% 18$. In our set up due to the limited resources and expertise available few patients with early stage disease are offered RFA treatment. Unfortunately in our study even among the very few patients with early stage HCC, only few were able to undergo ablative procedure due to poor performance status, lack of facilities and financial burdens. Nonsurgical treatment options are rather palliative. These include Transarterial Chemoembolization (TACE), Targeted therapy and chemotherapy.

The patients with child class $\mathrm{C}$ and thrombosis of portal vein cannot be offered TACE as it leads to hepatic failure and extrahepatic disease [19]. The two Pakistani studies have shown that TACE is the most commonly used treatment in HCC patients (60.7\% and 38.2\%) [6]. While these are the statistics from well-established tertiary care setups with overall survival of $45 \%$ at 5 years and $10 \%$ at 1 year [6]. While according to our study the results are even worse, as majority of our patients fell under child class B and C and most of them had portal vein thrombosis at the time of presentation so TACE was offered to only few of them. A targeted agent sorafenib is a tyrosine kinase inhibitor which is administered in the advanced stage and inoperable HCC patients. A study published by Cheng el al. in Asian patients sorafenib is considered as a first line therapy in patients who present in late stages and are not likely to benefit from TACE and ablative therapies [20]. Sorafenib is also indicated in the patients with Hepatitis B and C and alcohol induced HCC. Sorafenib has minimal benefit in late stage patients with median overall survival of 2.8 months [19]. Patients with child class $C$ having deranged clotting profile are not considered fit for targeted therapy due to increased risk of bleeding [20]. In our study patients were offered targeted therapy considering their stage of the disease, performance status, child class and affordability. Usually single agent chemotherapy drugs, doxorubicin and gemcitabine are employed in the treatment as compared to combination chemotherapy [19]. The therapeutic efficacy of both the drugs is similar [19]. In our study, single agent doxorubicin was offered in advanced stages only to the patients with good performance status and child class A. The external beam in the HCC has extremely limited role. The tolerance dose of liver is up to $30 \mathrm{~Gy}$ and normal hepatocytes are radiosensitive [21]. However in our study, the patients presented with spinal cord compression, bone and brain metastasis (total of $4.9 \%$ patients) were offered palliative hypofractionated external beam radiotherapy. The dosage protocols most frequently used in our setup are $30 \mathrm{~Gy}$ in 10 fractions, $20 \mathrm{~Gy}$ in 05 fractions and $08 \mathrm{~Gy}$ in single fraction.

\section{Conclusion and Recommendations}

$\mathrm{HCV}$ is the most common risk factor of hepatocellular carcinoma in Pakistan. The cirrhosis caused by HCV leads to the incurable form of HCC. However this menace can be controlled through prevention of Hepatitis C and early screening for hepatocellular carcinoma. Management plans should be discussed in multidisciplinary boards in the best benefit of the patients. More well-equipped and specialized liver centers should be established which should be made easily accessible to all the patients.

\section{References}

1. Schlageter M, Terracciano LM, D'Angelo S, Sorrentino P (2014) Histopathology of hepatocellular carcinoma. World J Gastroenterol 20 : 15955-15964.

2. Seeff LB (2004) Introduction: The burden of hepatocellular carcinoma. Gastroenterology 127 (5 Suppl 1): S1-S4.

3. El-Serag HB (2002) Hepatocellular carcinoma: an epidemiologic view. J Clin Gastroenterol. 35(5 Suppl 2): S72-S78.

4. Umar M, Bushra HT, Ahmad M, Data A, Ahmad M, et al. (2010) Hepatitis C in Pakistan: A Review of Available Data. Hepat Mon 10: 205-214.

5. Ali SA, Donahue RM, Qureshi H, Vermund SH (2009) Hepatitis B and hepatitis $\mathrm{C}$ in Pakistan: prevalence and risk factors. International journal of infectious diseases 13: 9-19.

6. Hafeez Bhatti AB, Dar FS, Waheed A, Shafique K, Sultan F, et al. (2016) Hepatocellular carcinoma in Pakistan: national trends and global perspective. Gastroenterol Res Pract 2016: 5942306.

7. Sangiovanni A, Del Ninno E, Fasani P, De Fazio C, Ronchi G, et al. (2004) Increased survival of cirrhotic patients with a hepatocellular carcinoma detected during surveillance. Gastroenterology 126: 1005-1014.

8. Choo SP, Tan WL, Goh BKP, Tai WM, Zhu AX (2016) Comparison of hepatocellular carcinoma in Eastern versus Western populations. Cancer 122: 3430-3446.

9. de Oliveria Andrade LJ, D'Oliveira A, Melo RC, De Souza EC, Costa Silva CA, et al. (2009) Association Between Hepatitis C and Hepatocellular Carcinoma. J Glob Infect Dis 1: 33-37.

10. Poon D, Anderson BO, Chen LT, Tanaka K, Lau WY, et al. (2009) Management of hepatocellular carcinoma in Asia: consensus statement from the Asian Oncology Summit 2009. Lancet Oncology 10: 1111-1118.

11. Colli A, Fraquelli M, Casazza G, Massironi S, Colucci A, et al. (2006) Accuracy of ultrasonography, spiral $\mathrm{CT}$, magnetic resonance, and alphafetoprotein in diagnosing hepatocellular carcinoma: a systematic review. Am J Gastroenterol 101: 513-523.

12. Colli A, Fraquelli M, Casazza G, Massironi S, Colucci A, et al. (2006) Accuracy of ultrasonography, spiral CT, magnetic resonance, and alphafetoprotein in diagnosing hepatocellular carcinoma: a systematic review. Am J Gastroenterol 101: 513-523.

13. Malaguarnera G, Paladina I, Giordano M, Malaguarnera M, Bertino G, et al (2013) Serum markers of intrahepatic cholangiocarcinoma. Dis Markers 34: 219-228.

14. Pons F, Varela M, Llovet JM (2005) Staging systems in hepatocellular carcinoma. HPB (Oxford) 7: 35-41.

15. Lin S, Hoffmann K, Schemmer P (2012) Treatment of hepatocellular carcinoma: a systematic review. Liver Cancer 1: 144-158.

16. Testa G, Crippin JS, Netto GJ, Goldstein RM, Jennings LW, et al. (200) Liver transplantation for hepatitis C: recurrence and disease progression in 300 
Citation: Fatima H, Mahmood H, Faheem M. Association of Hepatitis C with Hepatocelluar Carcinoma: A Cross-Sectional Study from Northern Pakistan. J Cancer Sci. 2018;5(1): 5.

patients. Liver Transpl 6: 553-561.

17. Molmenti EP, Klintmalm GB (2002) Liver transplantation in association with hepatocellular carcinoma: an update of the International Tumor Registry. Liver Transpl 8: 736-748.

18. Livraghi T, Meloni F, Di Stasi M, Rolle E, Solbiati L, et al. (2008) Sustained complete response and complications rates after radiofrequency ablation of very early hepatocellular carcinoma in cirrhosis: Is resection still the treatment of choice?. Hepatology 47: 82-89.
19. Lin S, Hoffmann K, Schemmer P (2012) Treatment of Hepatocellular Carcinoma: A Systematic Review. Liver Cancer 1: 144-158.

20. Cheng AL, Kang YK, Chen Z, Tsao CJ, Qin S, et al. (2009) Efficacy and safety of sorafenib in patients in the Asia-Pacific region with advanced hepatocellular carcinoma: a phase III randomised, double-blind, placebocontrolled trial. Lancet Oncol 10: 25-34.

21. Wharton JT, Delclos L, Gallager S, Smith JP (1973) Radiation hepatitis induced by abdominal irradiation with the cobalt 60 moving strip technique. Am J Roentgenol Radium Ther Nucl Med 117: 73-80.

\section{Acknowledgement}

I would like to thank Syed Muhammad Hassan Mustafa, Assistant Professor at National University of Computer and Emerging Sciences for his assistance with the statistics used in this research project. 